\title{
Theoretical analysis of conjugated heat transfer with a single domain formulation and integral transforms ${ }^{\text {tr }}$
}

\author{
Diego C. Knupp, Carolina P. Naveira-Cotta, Renato M. Cotta * \\ Laboratory of Transmission and Technology of Heat, LTTC, Mechanical Engineering Dept. - POLI E' COPPE, UFRJ, Universidade Federal do Rio de Janeiro, Cidade Universitária, \\ Cx. Postal 68503, Rio de Janeiro, RJ, CEP 21945-970 Brazil
}

\section{A R T I C L E I N F O}

Available online 9 January 2012

\section{Keywords:}

Conjugated heat transfer

Laminar channel flow

Single domain formulation

Integral transforms

Graetz problem

Internal convection

\begin{abstract}
A B S T R A C T
The present work advances an analytical approach for conjugated conduction-convection heat transfer problems, by proposing a single domain formulation for modeling both the fluid stream and the channel wall regions. Making use of coefficients represented as space variable functions with abrupt transitions occurring at the fluid-wall interface, the mathematical model is fed with the information concerning the transition of the two domains, unifying the model into a single domain formulation with space variable coefficients. The Generalized Integral Transform Technique (GITT) is then employed in the hybrid numerical-analytical solution of the resulting convection-diffusion problem with variable coefficients, and critically compared for two alternative solution paths. A test problem is chosen that offers an exact solution for validation purposes, based on the extended Graetz problem including transversal conduction across the channel walls. The excellent agreement between approximate and exact solutions demonstrates the feasibility of the approach in handling more involved conjugated problems.
\end{abstract}

(c) 2011 Elsevier Ltd. All rights reserved.

\section{Introduction}

The miniaturization of mechanical equipment such as heat exchangers is a subject of major interest in recent years, in light of the increasing demand on high performance devices and processes with high thermal efficiency, on sensors with rapid and accurate response, and on the ever growing need for heat dissipation in electronic devices, which tend to be conceived in even smaller dimensions and with more powerful data processing capacity.

For conception and design optimization, it is of crucial importance to employ reliable mathematical models and solution methodologies capable of describing the physical phenomena that take place in such micro-systems. Nevertheless, recent contributions have shown significant discrepancies between experimental results and macro-scale correlations and simulations $[1,2]$ which may be the result of neglecting terms that are usually not important at the macro-scale, but whose effects may have significant importance in micro-scale heat transfer. In order to achieve simulated results with better agreement against experimental data, a lot of effort is being dispended in the proposition of models and solution methodologies to deal with fluid flow and heat transfer in microchannels, such as the consideration of slip flow in opposition to the classical no-slip condition, the inclusion of terms related to the viscous dissipation and axial diffusion which are often neglected in macro-scale problems, besides the

\footnotetext{
is Communicated by W.J. Minkowycz.

* Corresponding author.

E-mail address: cotta@mecanica.ufrj.br (R.M. Cotta).
}

investigation of corrugated walls effects in heat transfer enhancement [3-11]. Recently, Nunes et al. [12], motivated by the theoretical conclusions reached in [13], presented some experimental and theoretical results showing the importance of taking into account the heat conduction within metallic microchannel walls, leading to a conjugated problem which solution yields results in much better agreement with the available experimental data. The theoretical approach then employed was an extension of the work of [14], based on the Generalized Integral Transform Technique (GITT), a hybrid numericalanalytical technique for the solution of convection-diffusion problems [15-20], accounting for the longitudinal heat conduction along the asymmetric walls.

The present work is thus aimed at progressing into the analysis of conjugated heat transfer in channels, developing and validating a methodology for the approximate treatment of the conjugated problem reformulated into a single domain model. Thus, inspired by the well succeeded approach developed in reference [21] for the solution of heat conduction problems in heterogeneous media, we propose in this paper the reformulation of conjugated problems as a single region model that accounts for the heat transfer at both the fluid flow and the channel wall regions. By making use of coefficients represented as space variable functions, with abrupt transitions occurring at the fluid-wall interface, the mathematical model is fed with the information concerning the two original domains of the problem.

For the solution of the proposed mathematical model we again make use of the Generalized Integral Transform Technique (GITT). This approach is based on extending the classical integral transform 


\begin{tabular}{|c|c|}
\hline \multicolumn{2}{|c|}{ Nomenclature } \\
\hline$u$ & fluid velocity; \\
\hline$T$ & temperature field; \\
\hline$y$ & transversal coordinate; \\
\hline$z$ & longitudinal coordinate; \\
\hline$w$ & heat capacity; \\
\hline$k$ & thermal conductivity; \\
\hline$y_{w}$ & $\begin{array}{l}\text { distance from the channel centerline to the external } \\
\text { face of the channel wall; }\end{array}$ \\
\hline$y_{i}$ & $\begin{array}{l}\text { distance from the channel centerline to the internal } \\
\text { face of the channel wall; }\end{array}$ \\
\hline Y & dimensionless transversal coordinate; \\
\hline$Z$ & dimensionless longitudinal coordinate; \\
\hline$U$ & dimensionless fully developed velocity profile; \\
\hline$\theta$ & dimensionless temperature field; \\
\hline$K$ & dimensionless thermal conductivity; \\
\hline $\mathrm{Pe}$ & Péclét number; \\
\hline $\mathrm{Nu}$ & Nusselt number; \\
\hline$N$ & Truncation order of the temperature field expansions; \\
\hline M & Truncation order of the auxiliary problem, Eqs. $(14 a, b)$ \\
\hline
\end{tabular}

\section{Greek letters}

$\psi \quad$ eigenfunction of the constant coefficients eigenvalue problem;

$\xi \quad$ eigenfunction of the space variable coefficients eigenvalue problem;

$\Omega \quad$ eigenfunction of the auxiliary problem corresponding to the variable coefficients eigenvalue problem; $\phi \quad$ eigenfunction of the exact solution;

$\mu, \beta, \lambda, \gamma$ eigenvalues corresponding to $\psi, \xi, \Omega$ and $\phi$, respectively;

\section{Subscripts \& Superscripts}

$i, n \quad$ order of eigenquantities

- $\quad$ integral transform

normalized eigenfunction

* $\quad$ filtered temperature field

$s \quad$ quantity corresponding to the solid region (channel walls)

$f \quad$ quantity corresponding to the fluid flow region

in quantity corresponding to the entrance of the channel $(Z=0)$

$w$ quantity corresponding to the external face of the channel wall

method [22], making it sufficiently flexible to handle problems that are not a priori transformable, such as in the case of problems with arbitrarily space-dependent and nonlinear coefficients in either the equation or the boundary conditions. In order to validate the approximate solution herein obtained, a test problem was chosen based on an extended Graetz problem with transversal conduction across the wall, which results in an exact solution for the conjugated problem achieved with the Classical Integral Transform Technique (CITT), here used as a benchmark result. The developed approach is critically compared for two alternative solution paths. First, we propose the simplest possible auxiliary problem with constant coefficients to demonstrate this most flexible solution path via GITT. Then, the auxiliary problem is formulated by directly applying separation of variables to the homogeneous version of the original problem so that all the information concerning the transition of the two domains is represented by the space variable coefficients of the eigenvalue problem, which in this case is tackled with the GITT itself.

\section{Problem formulation and solution methodology}

The problem chosen to illustrate the proposed methodology involves a laminar incompressible internal flow of a Newtonian fluid between parallel plates, in steady-state and undergoing convective heat transfer due to a prescribed temperature, $T_{w}$, at the external face of the channel wall. The channel wall is considered to participate on the heat transfer problem through transversal heat conduction only. The fluid flows with a known fully developed velocity profile $u_{f}(y)$, and with an inlet temperature $T_{\text {in. }}$. Fig. 1 depicts a schematic representation of this problem.

\subsection{Single domain formulation}

We assume that the flow is dynamically developed and thermally developing. Then, the formulation of the conjugated problem as a single region model that accounts for the heat transfer phenomena at both the fluid flow and the channel solid wall is achieved by making use of coefficients represented as space variable functions where the abrupt transitions occur at the fluid-solid wall interface. The conjugated problem is then given by the following single domain formulation with space variable coefficients:

$u(y) w_{f} \frac{\partial T(y, z)}{\partial z}=k(y) \frac{\partial^{2} T}{\partial z^{2}}+\frac{\partial}{\partial y}\left(k(y) \frac{\partial T}{\partial y}\right), 0<y<y_{w}, z>0$

$T(y, z=0)=T_{\text {in }}$

$\left.\frac{\partial T}{\partial y}\right|_{y=0}=0, T\left(y=y_{w}, z\right)=T_{w}$

where the following space variable functions have been used:

$u(y)=\left\{\begin{array}{cc}u_{f}(y), & \text { if } 0<y<y_{i} \\ 0, & \text { if } y_{i}<y<y_{w}\end{array}\right.$
$k(y)=\left\{\begin{array}{cc}k_{f}, & \text { if } 0<y<y_{i} \\ k_{s}, & \text { if } y_{i}<y<y_{w}\end{array}\right.$

where $w_{f}$ is the heat capacity of the fluid, $k_{s}$ is the thermal conductivity of the channel wall, $k_{f}$ is the thermal conductivity of the fluid, and $u_{f}(y)$ is the known parabolic velocity profile of the fully developed flow. Defining the following dimensionless groups:

$$
\begin{aligned}
& Z=\frac{z / y_{w}}{\operatorname{RePr}}=\frac{z}{y_{w} \operatorname{Pe}} ; Y=\frac{y}{y_{w}} ; U=\frac{u}{4 u_{a v}} ; \theta=\frac{T-T_{i n}}{T_{w}-T_{i n}} ; K=\frac{k}{k_{f}} \\
& \operatorname{Re}=\frac{u_{a v} 4 y_{w}}{v} ; \operatorname{Pr}=\frac{v}{\alpha} ; \operatorname{Pe}=\operatorname{RePr}=\frac{u_{a v} 4 y_{w}}{\alpha} ; \alpha=\frac{k_{f}}{w_{f}}
\end{aligned}
$$

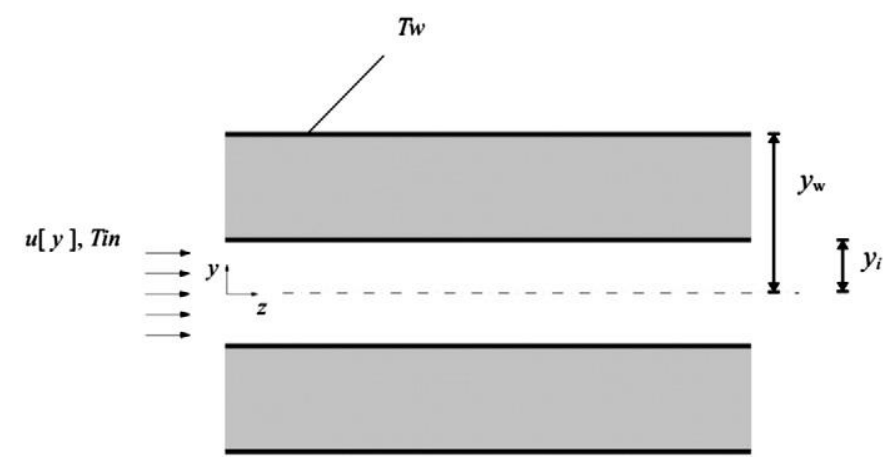

Fig. 1. Schematic representation of the conjugated heat transfer problem. 
we obtain the dimensionless formulation of this problem

$U(Y) \frac{\partial \theta(Y, Z)}{\partial Z}=\frac{K(Y)}{P^{2}} \frac{\partial^{2} \theta}{\partial Z^{2}}+\frac{\partial}{\partial Y}\left(K(Y) \frac{\partial \theta}{\partial Y}\right), 0<Y<1, Z>0$

$\theta(Y, Z=0)=0$

$\left.\frac{\partial \theta}{\partial Y}\right|_{Y=0}=0, \quad \theta(Y=1, Z)=1$

where

$U(Y)=\left\{\begin{array}{cc}U_{f}(Y), & \text { if } 0<Y<Y_{i}=y_{i} / y_{w} \\ 0, & \text { if } Y_{i}<Y<1\end{array}\right.$

$K(Y)=\left\{\begin{array}{cc}1, & \text { if } 0<Y<Y_{i}=y_{i} / y_{w} \\ k_{s} / k_{f}, & \text { if } Y_{i}<Y<1\end{array}\right.$

To improve the computational performance of the formal solutions to be derived below, it is recommended to reduce the importance of the boundary source terms, so as to enhance the eigenfunction expansions convergence behavior [18]. One possible approach for achieving this goal is the proposition of analytical filtering solutions, and in this work the proposed filter is just the temperature at the external wall, as represented in the following expression:

$\theta(Y, Z)=1+\theta^{*}(Y, Z)$

The filtered problem is thus rewritten by substituting Eq. (4) into Eqs. (3a)-(3f).

For validation purposes, in order to obtain a problem that still offers a fairly simple exact solution, we neglect the axial conduction term in Eq. (3a), which is a feasible assumption for this problem when dealing with high Péclét numbers and walls with a low thermal conductivity, such as micro-channel walls made of polymeric materials. The following equations provide the filtered problem formulation when the axial conduction term is neglected:

$U(Y) \frac{\partial \theta^{*}(Y, Z)}{\partial Z}=\frac{\partial}{\partial Y}\left(K(Y) \frac{\partial \theta^{*}}{\partial Y}\right), 0<Y<1, Z>0$

$\theta^{*}(Y, Z=0)=\theta_{Z=0}^{*}=-1$

$\left.\frac{\partial \theta^{*}}{\partial Y}\right|_{Y=0}=0, \theta^{*}(Y=1, Z)=0$

Two alternatives for handling problem (5) above via integral transforms are now more closely considered. The first methodology involves the consideration of an auxiliary eigenvalue problem with constant coefficients, thus without transferring to the eigenfunction expansion basis the information on the space variation of the original problem coefficients. Although this could be the simplest and most flexible solution path when employing the GITT, it is not necessarily the most effective from the computational point of view, as we shall examine within the results and discussion section. The second methodology is based on considering the auxiliary eigenvalue problem with all the information on the space variable coefficients, as obtained from applying separation of variables to the homogeneous version of the originally posed problem. In this case, the GITT itself must eventually be employed in the solution of the proposed eigenvalue problem, but may also result in marked simplification of the following steps in the solution methodology, with an overall gain in convergence rates and computational efficiency, as we shall later on discuss.

\subsection{Solution with constant coefficients eigenvalue problem}

Following the GITT formalism, the transform/inverse pair for solving problem (5) with a constant coefficients eigenvalue problem is defined as follows:

transform : $\bar{\theta}_{n}^{*}(Z)=\int_{0}^{1} \tilde{\psi}_{n}(Y) \theta^{*}(Y, Z) d Y$

inverse : $\theta^{*}(Y, Z)=\sum_{n=1}^{\infty} \tilde{\psi}_{n}(Y) \bar{\theta}_{n}^{*}(Z)$

where

$\tilde{\psi}_{n}(Y)=\frac{\psi_{n}(Y)}{\sqrt{N_{n}}}$, normalized eigenfunctions

$N_{n}=\int_{0}^{1} \psi_{n}^{2}(Y) d Y$, normalization integrals

where the eigenfunctions $\psi_{n}(Y)$ come from the eigenvalue problem solution, which is here first chosen as the simplest possible auxiliary problem, to demonstrate this flexible solution path which is very attractive for implementation in automatic solvers such as the recently developed UNIT code (UNified Integral Transforms) [23]:

$\frac{d^{2} \psi_{n}(Y)}{d Y^{2}}+\mu_{n}{ }^{2} \psi_{n}(Y)=0$

$\left.\frac{d \psi_{n}}{d Y}\right|_{Y=0}=0, \quad \psi_{n}(Y=1)=0$

Operating Eq. (5a) on with $\int_{0}^{1} \tilde{\psi}_{n}(Y)(\cdot) d Y$, one obtains:

$$
\begin{aligned}
\int_{0}^{1} U(Y) \frac{\partial \theta^{*}}{\partial Z} \tilde{\psi}_{n}(Y) d Y & =\int_{0}^{1} \tilde{\psi}_{n}(Y) \frac{\partial}{\partial Y}\left(K(Y) \frac{\partial \theta^{*}}{\partial Y}\right) d Y= \\
& =\int_{0}^{1} \frac{\partial}{\partial Y}\left(\tilde{\psi}_{n}(Y) K(Y) \frac{\partial \theta^{*}}{\partial Y}\right) d Y-\int_{0}^{1} \frac{d \tilde{\psi}_{n}(Y)}{d Y} K(Y) \frac{\partial \theta^{*}}{\partial Y} d Y
\end{aligned}
$$

which can be rewritten as:

$\left.\int_{0}^{1} U(Y) \frac{\partial \theta^{*}}{\partial Z} \tilde{\psi}_{n}(Y) d Y=\tilde{\psi}_{n}(Y) K(Y) \frac{\partial \theta^{*}}{\partial Y}\right)_{0}^{1}-\int_{0}^{1} \frac{d \tilde{\psi}_{n}(Y)}{d Y} K(Y) \frac{\partial \theta^{*}}{\partial Y} d Y$

where

$\left.\tilde{\psi}_{n}(Y) K(Y) \frac{\partial \theta^{*}}{\partial Y}\right)_{0}^{1}=0$

Thus:

$\int_{0}^{1} U(Y) \frac{\partial \theta^{*}}{\partial Z} \tilde{\psi}_{n}(Y) d Y=-\int_{0}^{y_{w}} \frac{d \tilde{\psi}_{n}(Y)}{d Y} k(Y) \frac{\partial \theta^{*}}{\partial Y} d Y$

Using the inverse formula (6b) into Eq. (8d), one obtains:

$\int_{0}^{1} U(Y)\left[\sum_{m=1}^{\infty} \frac{d \bar{\theta}_{m}^{*}}{d Z} \tilde{\psi}_{m}(Y)\right] \tilde{\psi}_{n}(Y) d Y=-\int_{0}^{1} \frac{d \tilde{\psi}_{n}(Y)}{d Y} K(Y)\left[\sum_{m=1}^{\infty} \bar{\theta}_{m}^{*} \frac{d \tilde{\psi}_{m}(Y)}{d Y}\right] d Y$

which can be rewritten as:

$\sum_{m=1}^{\infty} \frac{d \bar{\theta}_{m}^{*}}{d Z} \int_{0}^{1} U(Y) \tilde{\psi}_{n}(Y) \tilde{\psi}_{m}(Y) d Y=-\sum_{m=1}^{\infty} \bar{\theta}_{m}^{*} \int_{0}^{1} K(Y) \frac{d \tilde{\psi}_{n}(Y)}{d Y} \frac{d \tilde{\psi}_{m}(Y)}{d Y} d Y$ 
and concisely given in matrix form by:

$$
\mathbf{A} \frac{d \bar{\theta}^{*}}{d Z}=-\mathbf{B} \bar{\theta}^{*}
$$

$$
\text { where } \begin{aligned}
A_{n m} & =\int_{0}^{1} U(Y) \tilde{\psi}_{n}(Y) \tilde{\psi}_{m}(Y) d Y, \\
B_{n m} & =\int_{0}^{1} K(Y) \frac{d \tilde{\psi}_{n}(Y)}{d Y} \frac{d \tilde{\psi}_{m}(Y)}{d Y} d Y
\end{aligned}
$$

The ordinary differential equations (ODE) system (9) can be analytically solved to provide results for the transformed temperatures, upon truncation to a sufficiently large finite order $\mathrm{N}$, in terms of the matrix exponential function

$\underline{\tilde{\theta}}^{*}(Z)=\exp \left(-\mathbf{A}^{-1} \mathbf{B} Z\right) \underline{\theta}^{*}(0)$

where $\bar{\theta}^{*}(0)$ are the transformed initial conditions, given by:

$\bar{\theta}_{n}^{*}(0)=\int_{0}^{1} \tilde{\psi}_{n}(Y) \theta_{Z=0}^{*} d Y$

Once the transformed potentials $\bar{\theta}_{n}^{*}(Z)$, with $n=1,2, \ldots, N$, have been computed, the inversion formula can be recalled to yield the temperature field $\theta^{*}(Y, Z)$ representation at any desired position $Y$ and $Z$. The original dimensionless temperature field $\theta(Y, Z)$ can then be approximately obtained in analytical form by:

$\theta(Y, Z)=1+\theta^{*}(Y, Z)=1+\sum_{n=1}^{N} \bar{\theta}_{n}^{*}(Z) \tilde{\psi}_{n}(Y)$

\subsection{Solution with Variable Coefficients Eigenvalue Problem}

In many applications, especially when dealing with inverse or optimization problems, it becomes crucial to adopt a solution methodology that is both accurate and computationally fast, so as to allow for intensive iterative analyses. In this context, it is desirable that the eigenvalue problem be chosen in order to contain as much information as possible about the original problem. The following eigenvalue problem has been formulated by directly applying separation of variables to problem (5) so that all the information concerning the transition of the two domains is represented within the eigenvalue problem, by means of the space variable coefficients $K(Y)$ and $U(Y)$. Thus,

$\frac{d}{d Y}\left(K(Y) \frac{d \zeta_{i}(Y)}{d Y}\right)+\beta_{i}^{2} \zeta_{i}(Y) U(Y)=0$

$\left.\frac{d \zeta_{i}}{d Y}\right|_{Y=0}=0, \quad \zeta_{i}(Y=1)=0$

Problem (12) does not allow for an explicit analytic solution, but the GITT itself can be used in order to provide a hybrid numericalanalytical solution. The GITT is here employed in the solution of this eigenvalue problem via the proposition of a simpler auxiliary eigenvalue problem, and expanding the unknown eigenfunctions in terms of the chosen basis. The chosen auxiliary problem is given by:

$\frac{d^{2} \Omega_{n}(Y)}{d Y^{2}}+\lambda_{n}^{2} \Omega_{n}(Y)=0$

$$
\left.\frac{d \Omega_{n}(Y)}{d Y}\right|_{Y=0}=0, \Omega_{n}(Y=1)=0
$$

The proposed expansion of the original eigenfunction is then given by:

$\zeta_{i}(Y)=\sum_{n=0}^{\infty} \tilde{\Omega}_{n}(Y) \bar{\zeta}_{i, n}, \quad$ inverse

$\bar{\zeta}_{i, n}=\int_{0}^{1} \zeta_{i}(Y) \tilde{\Omega}_{n}(Y) d Y$, transform

The integral transformation of the eigenvalue problem with space variable coefficients is then performed by operating on Eq. (5a) with $\int_{0}^{1} \tilde{\Omega}_{n}(Y)(\cdot) d Y$, to yield the following algebraic problem in matrix form [21]:

$$
(\mathbf{A}-\nu \mathbf{B}) \bar{\zeta}=0, \text { with } \nu_{i}=\beta_{i}^{2}
$$

$\bar{\zeta}=\left\{\bar{\zeta}_{n, m}\right\} ; \mathbf{B}=\left\{B_{n, m}\right\}, B_{n, m}=\int_{0}^{1} U(Y) \tilde{\Omega}_{n}(Y) \tilde{\Omega}_{m}(Y) d Y$

$$
\begin{aligned}
\mathbf{A}=\left\{A_{n, m}\right\} ; A_{n, m} & =\int_{0}^{1} \tilde{\Omega}_{m}(Y) \frac{d}{d Y}\left(K(Y) \frac{d \tilde{\Omega}_{n}(Y)}{d Y}\right) d Y= \\
& =-\int_{0}^{1} K(Y) \frac{d \tilde{\Omega}_{m}(Y)}{d Y} \frac{d \tilde{\Omega}_{n}(Y)}{d Y} d Y
\end{aligned}
$$

The algebraic problem (15a) can be numerically solved to provide results for the eigenvalues and eigenvectors, upon truncation to a sufficiently large finite order $\mathrm{M}$, and then combined by the inverse formula (9a) to provide the desired original eigenfunctions.

Once the solution of the eigenvalue problem (12) is made available, the original problem (5) becomes completely transformable and the final solution is then obtainable by separation of variables, and given by:

$$
\begin{aligned}
& \theta(Y, Z)=1+\theta^{*}(Y, Z)=1+\sum_{i=1}^{N} \bar{\theta}_{Z=0, i}^{*} \exp \left(-\beta_{i}^{2} Z\right) \tilde{\xi}_{i}(Y) \\
& \bar{\theta}_{Z=0, i}^{*}=\int_{0}^{1} U(Y) \tilde{\xi}_{i}(Y) \theta_{Z=0}^{*} d Y
\end{aligned}
$$

\subsection{Exact Solution}

Problem (5) has been here selected for illustration of the methodology since a straightforward exact solution can be readily obtained. For this purpose, the heat transfer problem is then modeled as a conduction problem for the solid wall, coupled at the interface $Y=Y_{i}$ with the internal convective problem for the fluid, as represented by the following equations:

Solid heat conduction equation:

$0=\frac{\partial^{2} \theta_{s}(Y, Z)}{\partial Y^{2}}, Y_{i}<Y<1, Z>0$

$\theta_{s}(Y=1, Z)=1$

Fluid heat convection equation:

$U_{f}(Y) \frac{\partial \theta_{f}(Y, Z)}{\partial Z}=\frac{\partial^{2} \theta_{f}}{\partial Y^{2}}, 0<Y<Y_{i}, Z>0$

$\theta_{f}(Y, Z=0)=0$

$\left.\frac{\partial \theta_{f}}{\partial Y}\right|_{Y=0}=0$ 
Interface conditions:

$\left.\frac{\partial \theta_{f}}{\partial Y}\right|_{Y=Y_{i}}=\left.\frac{k_{s}}{k_{f}} \frac{\partial \theta_{s}}{\partial Y}\right|_{Y=Y_{i}}$ and $\theta_{f}\left(Y_{i}, Z\right)=\theta_{s}\left(Y_{i}, Z\right)$

For the exact solution of the proposed problem, Eqs. (17), we first consider Eq. (17a) and the boundary conditions given by Eqs. (17b) and $(17 \mathrm{~g})$, which readily yield the following expression for the solid wall temperature distribution:

$\theta_{s}(Y, Z)=1-\frac{1-\theta_{f}\left(Y_{i}, Z\right)}{1-Y_{i}}+\frac{1-\theta_{f}\left(Y_{i}, Z\right)}{1-Y_{i}} Y$

and the boundary condition given by Eq. (17f) can then be rewritten as:

$\left.\frac{\partial \theta_{f}}{\partial Y}\right|_{Y=Y_{i}}+\frac{k_{s}}{k_{f}} \frac{1}{1-Y_{i}} \theta_{f}\left(Y_{i}, Z\right)=\frac{k_{s}}{k_{f}} \frac{1}{1-Y_{i}}$

Thus, the problem for the fluid flow region becomes a Graetz type problem with third kind boundary condition:

$U_{f}(Y) \frac{\partial \theta_{f}(Y, Z)}{\partial Z}=\frac{\partial^{2} \theta_{f}}{\partial Y^{2}}, 0<Y<Y_{i}, Z>0$

$\theta_{f}(Y, Z=0)=0$

$\left.\frac{\partial \theta_{f}}{\partial Y}\right|_{Y=0}=0,\left.\quad \frac{\partial \theta_{f}}{\partial Y}\right|_{Y=Y_{i}}+\frac{k_{s} / k_{f}}{1-Y_{i}} \theta_{f}\left(Y_{i}, Z\right)=\frac{k_{s} / k_{f}}{1-Y_{i}}$

Problem (20) has an exact analytical solution readily obtainable by the Classical Integral Transform Technique [16,22] and then the channel wall region temperature distribution, $\theta_{s}(Y, Z)$, can be directly obtained from Eq. (18). This exact solution for the wall and fluid flow regions will later on be used as a benchmark solution for the validation of the conjugated problem approximate solutions described in the previous sections. The exact solution for the fluid flow region is obtained from the solution of the following eigenvalue problem, formulated by directly applying separation of variables to the homogeneous version of problem (20):

$\frac{d^{2} \phi(Y)}{d Y^{2}}+U_{f}(Y) \gamma^{2} \phi(Y)=0$

$\left.\frac{d \phi}{d Y}\right|_{Y=0}=0,\left.\quad \frac{d \phi}{d Y}\right|_{Y=Y_{i}}+\frac{k_{s} / k_{f}}{1-Y_{i}} \phi\left(Y_{i}\right)=0$

which allows for an analytical solution in terms of hypergeometric functions that can be readily obtained using the routine DSolve of the Mathematica platform [6].

\subsection{Nusselt Number Calculation}

The main interest in convective heat transfer analysis is often to determine the local Nusselt number, $N u(Z)$, to be evaluated here from both the approximate and the exact solutions. The following expressions for the local Nusselt number and for the bulk temperature are then employed:

$N u(Z)=-\left.\frac{1}{\theta_{a v}(Z)} \frac{\partial \theta(Y, Z)}{\partial Y}\right|_{Y=Y_{i}}, \quad \theta_{a v}(Z)=\frac{\int_{0}^{Y_{i}} U_{f}(Y) \theta(Y, Z) d Y}{\int_{0}^{1} U(Y) d Y}$

In order to avoid the direct evaluation of the derivative $\partial \theta /\left.\partial Y\right|_{Y=Y_{i}}$ when using the approximate solutions, the following alternative integral balance formula [18] has been used:

$\int_{0}^{Y_{i}} U(Y) \frac{\partial \theta}{\partial Z} d Y=\int_{0}^{Y_{i}} \frac{\partial}{\partial Y}\left(\frac{\partial \theta}{\partial Y}\right) d Y$, or $\left.\frac{\partial \theta}{\partial Y}\right|_{Y=Y i}=\int_{0}^{Y_{i}} U(Y) \frac{\partial \theta}{\partial Z} d Y$

\section{Results and Discussion}

Fig. 2a,b below illustrate the behavior of the space variable coefficients that are feeding the single region model in Eq. (3a), $U(Y)$ and $K(Y)$, as space variable functions where the region from $Y=0$ to $Y=Y_{i}=0.5$ corresponds to the fluid flow domain and the region from $Y=Y_{i}=0.5$ to $Y=1$ corresponds to the channel wall. The dimensionless thermal conductivity has been calculated motivated by an application with a microchannel made of polyester resin $\left(k_{s}=0.16 \mathrm{~W} / \mathrm{m}^{\circ} \mathrm{C}\right)$ with water as the working fluid $\left(k_{f}=0.64 \mathrm{~W} / \mathrm{m}^{\circ} \mathrm{C}\right)$, so that $k_{s} / k_{f}=0.25$ [24].

The conjugated problem presented in this work has been solved using the approximate single region approach described in Sections 2.1-2.3 and compared to the exact solution of Section 2.4. Fig. 3a shows the transversal temperature profiles for a few different longitudinal positions along the flow, $Z=0.01,0.05,0.1,0.2,0.3,0.5$, $0.75,1.0$ and 1.5 , for the fluid and the channel wall regions obtained with the solution with the constant coefficients eigenvalue problem described in Section 2.2, with $\mathrm{N}=20$ terms as the truncation order. In these results it can be observed the excellent agreement between the approximate and exact solutions, which are essentially coincident to the graph scale. In Fig. $3 \mathrm{~b}$ it can be seen the temperature evolution at the centerline of the channel $(Y=0)$ for $Z=0$ up to 1.6, again using the single domain approach with constant coefficients eigenvalue problem, and it also shows the Graetz problem solution with first kind boundary condition, which is a simplification of this problem when the wall thermal resistance is neglected. In this test case, it can be concluded that the thermal resistance of the polymeric wall noticeably delays the increase of the fluid temperature along the flow.

Tables 1a, 1b illustrate the convergence behavior of the temperature profile for the single domain approach with constant coefficients eigenvalue problem (Section 2.2), respectively at $Z=0.01$ and $Z=0.05$, for different positions in the fluid flow region. The results are apparently fully converged to at least three digits for $\mathrm{N}=50$ in all selected positions. The exact solution results are fully converged to all five digits shown, which are achieved to within only five terms in the eigenfunction expansion. One may observe that in all selected positions the error of the approximate solution with respect to the exact one was smaller than $1.26 \%$, which occurred at the interface position for $Z=0.01$.

The approximate solution for the single domain approach with variable coefficients eigenvalue problem, here proposed in Section 2.3, is also critically examined. First, Table 2 illustrates the excellent convergence behavior of the first 10 eigenvalues associated with the original problem, Eqs. (12), and Fig. 4 depicts the convergence behavior of the 10th eigenfunction, for different truncation orders in Eq. (14a), $\mathrm{M}=10,12,14$, and 16 , where it can be noticed that with only 16 terms the 10th eigenfunction is fully converged to the graph scale.

The comparison of both approximate solutions with the exact solution at $Z=0.01$ is shown in Table 3 , in which the single domain approach with variable coefficients eigenvalue problem has been 

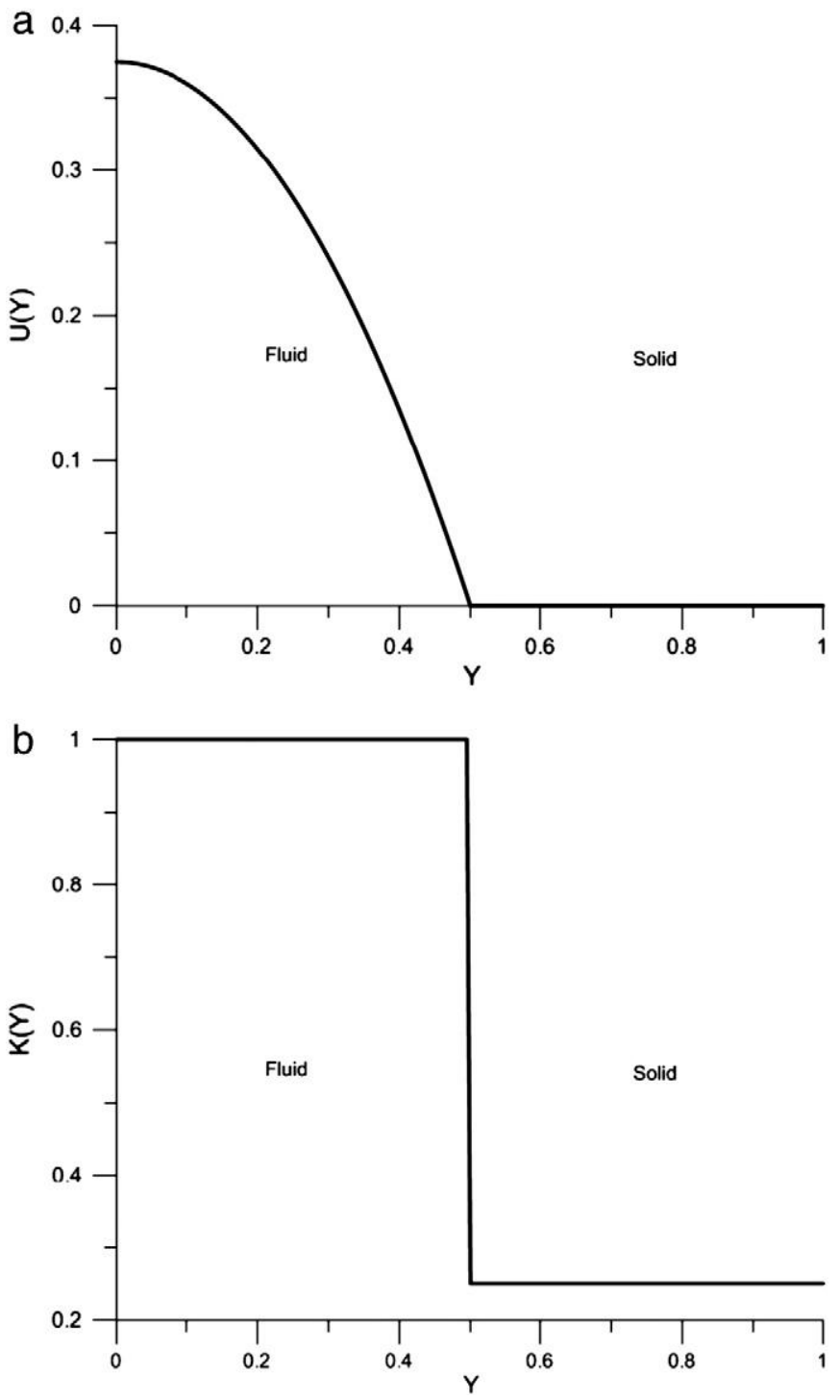

Fig. 2. a Representation of the space variable coefficients as space variable functions with the abrupt transition occurring at the interface fluid-solid wall: $U(Y)$.b Representation of the space variable coefficients as space variable functions with the abrupt transition occurring at the interface fluid-solid wall: $K(Y)$.

obtained with $\mathrm{M}=50$ terms in the eigenvalue problem solution and $\mathrm{N}=5$ terms in the temperature expansion, achieving full convergence to the five digits shown. It can be noticed that the relative error dropped significantly with the variable coefficients approach, which is computationally faster and more adequate for inverse and optimization problems. Fig. 5 finally depicts both the approximate and the exact computations of the local Nusselt number, where an excellent agreement can also be observed. It is also clear that the thermal development in the case under consideration, with the establishment of a fully developed asymptotic Nusselt number, is noticeably delayed with respect to the classical Graetz problem with prescribed wall temperature (case without wall conjugation). It is also evident that marked differences between the estimates of Nusselt number, with and without wall conjugation, can be achieved.

\section{Conclusions}

A single domain approach has been developed and validated for the approximate analytical treatment of conjugated heat transfer problems, here illustrated for laminar thermally developing channel flow, modeling the heat transfer phenomena at both the fluid flow
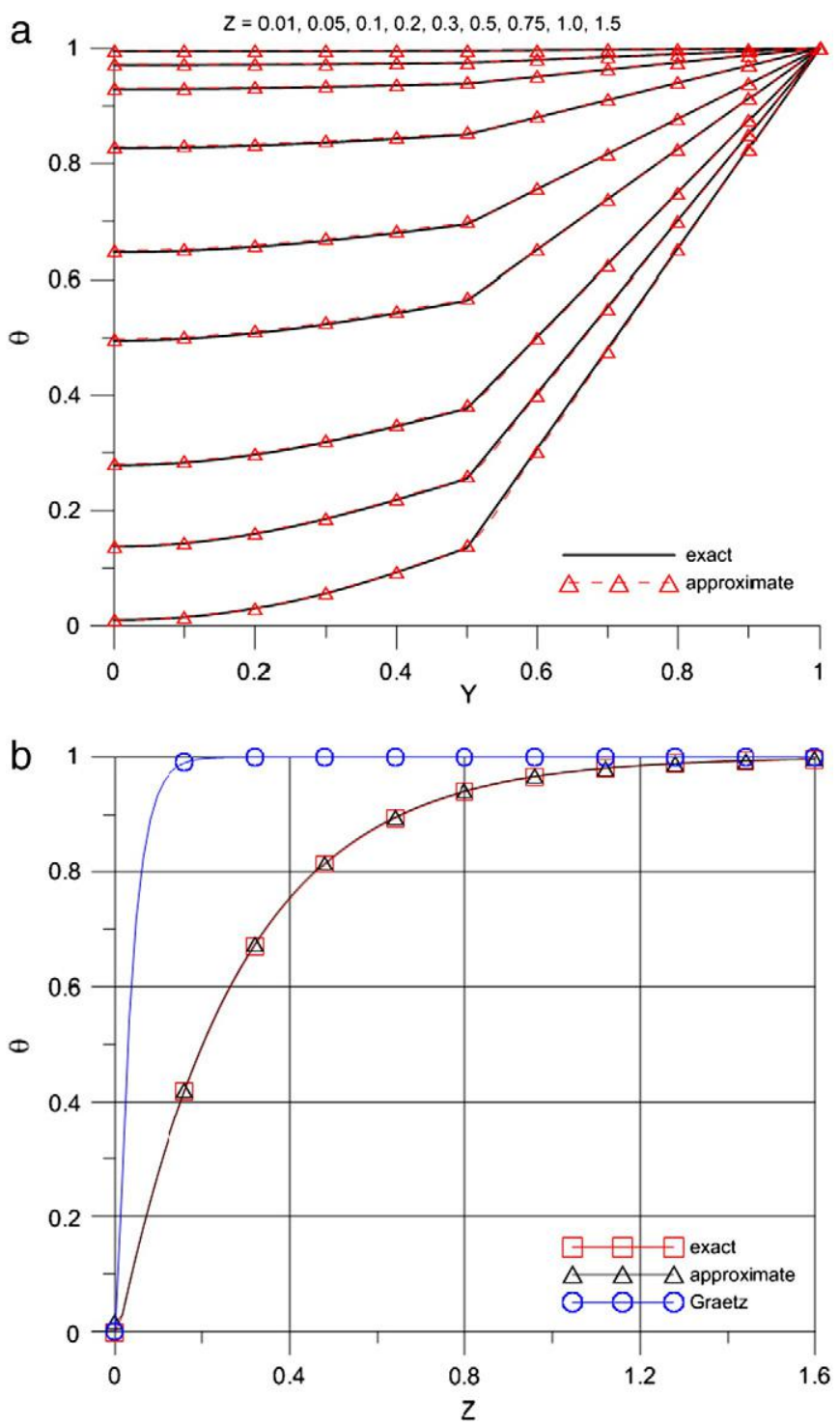

Fig. 3. a Temperature profiles calculated using the single domain approach with constant coefficients eigenvalue problem in comparison with the exact solution.b Comparison of the temperature evolution along the centerline of the channel $(Y=0)$ for $Z=0$ up to 1.6, using the single domain approach with constant coefficients eigenvalue problem in comparison with the exact solution and Graetz problem solution with first kind boundary condition.

and the channel wall regions. By making use of coefficients represented as space variable functions, with abrupt transitions occurring at the fluid-channel wall interface, the mathematical model is fed with the information concerning both domains of the problem. The chosen test problem has been tackled with the Generalized Integral Transform Technique (GITT), with both a straightforward constant coefficients eigenvalue problem and a more elaborate space variable

Table 1a

Convergence behavior of the temperature profile for the single domain approach with constant coefficients eigenvalue problem, at $Z=0.01$, for the fluid flow region.

\begin{tabular}{llll}
\hline order & $Y=0$ & $Y=0.25$ & $Y=0.5$ \\
\hline $\mathrm{N}=10$ & 0.010441 & 0.041455 & 0.14716 \\
$\mathrm{~N}=20$ & 0.010389 & 0.042408 & 0.14068 \\
$\mathrm{~N}=30$ & 0.010507 & 0.042456 & 0.13856 \\
$\mathrm{~N}=40$ & 0.010482 & 0.042288 & 0.13754 \\
$\mathrm{~N}=50$ & 0.010500 & 0.042329 & 0.13704 \\
Exact solution & 0.010413 & 0.042193 & 0.13534 \\
Relative error & $0.84 \%$ & $0.32 \%$ & $1.26 \%$ \\
\hline
\end{tabular}


Table 1b

Convergence behavior of the temperature profile for the single domain approach with constant coefficients eigenvalue problem, at $Z=0.05$, for the fluid flow region.

\begin{tabular}{llll}
\hline order & $Y=0$ & $Y=0.25$ & $Y=0.5$ \\
\hline $\mathrm{N}=10$ & 0.13810 & 0.17176 & 0.26606 \\
$\mathrm{~N}=20$ & 0.13806 & 0.17258 & 0.26048 \\
$\mathrm{~N}=30$ & 0.13816 & 0.17262 & 0.25865 \\
$\mathrm{~N}=40$ & 0.13807 & 0.17247 & 0.25778 \\
$\mathrm{~N}=50$ & 0.13815 & 0.17251 & 0.25735 \\
Exact solution & 0.13764 & 0.17195 & 0.25547 \\
Relative error & $0.37 \%$ & $0.33 \%$ & $0.74 \%$ \\
\hline
\end{tabular}

Table 2

Convergence behavior of the first ten eigenvalues in problem (12).

\begin{tabular}{lllll}
\hline Eigenvalue $\beta_{i}$ & $\mathrm{~N}=30$ & $\mathrm{~N}=60$ & $\mathrm{~N}=90$ & $\mathrm{~N}=120$ \\
\hline 1 & 1.89403 & 1.89112 & 1.89014 & 1.88965 \\
2 & 14.3682 & 14.3671 & 14.3667 & 14.3665 \\
3 & 27.3581 & 27.3574 & 27.3571 & 27.3570 \\
4 & 40.3901 & 40.3896 & 40.3894 & 40.3893 \\
5 & 53.4355 & 53.4352 & 53.4350 & 53.4349 \\
6 & 66.4872 & 66.4869 & 66.4868 & 66.4867 \\
7 & 79.5423 & 79.5421 & 79.5420 & 79.5419 \\
8 & 92.5995 & 92.5994 & 92.5993 & 92.5992 \\
9 & 105.658 & 105.658 & 105.658 & 105.658 \\
10 & 118.718 & 118.718 & 118.718 & 118.718 \\
\hline
\end{tabular}

coefficients case, in which the auxiliary problem itself is handled by GITT. An excellent agreement between the approximate and exact solutions was obtained, demonstrating the feasibility of the general approach herein proposed. It was also observed that the solution path that accounts for the space variable behavior within the eigenfunction expansion basis, provides better convergence rates and more efficient computational performance, in comparison to the solution with an eigenvalue problem with constant coefficients, and should in principle be preferred when dealing with more involved conjugated problems.

\section{Acknowledgements}

The authors would like to acknowledge financial support provided by the Brazilian agencies CNPq, FAPERJ and CAPES.

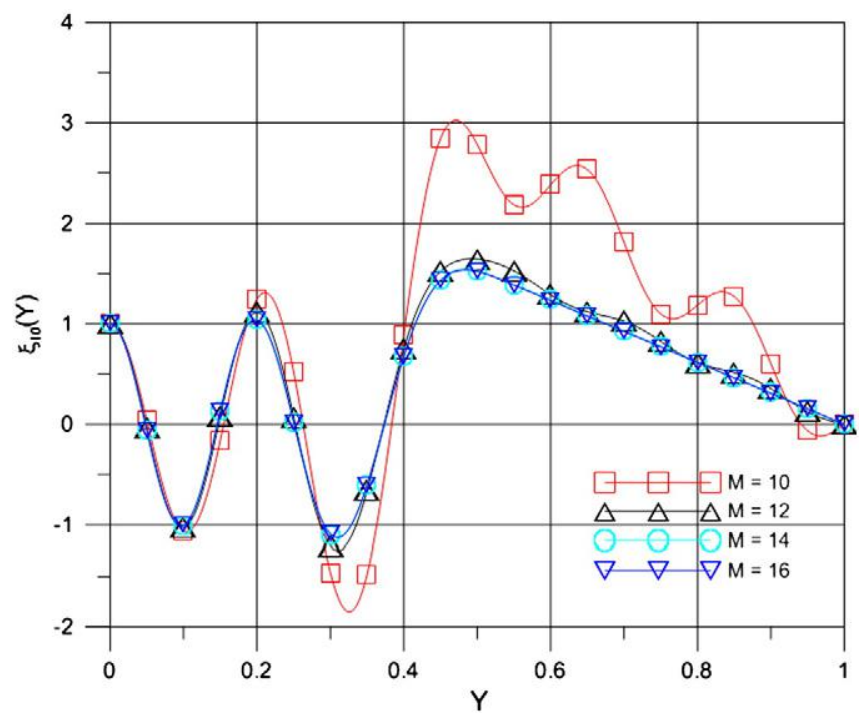

Fig. 4. Convergence behavior of the 10th eigenfunction of problem (12), for different truncation orders in Eq. (14a), $\mathrm{M}=10,12,14$, and 16 .
Table 3

Comparison between the approximate solutions (Sections 2.2 and 2.3) and the exact solution for the temperature at $Z=0.01$.

\begin{tabular}{llllll}
\hline$Y$ & $\begin{array}{l}\text { Approx. sol. } \\
\text { (Section 2.2) }\end{array}$ & $\begin{array}{l}\text { Approx. sol. } \\
\text { (Section 2.3) }\end{array}$ & Exact sol. & $\begin{array}{l}\text { Relative error } \\
\text { (Section 2.2) }\end{array}$ & $\begin{array}{l}\text { Relative error } \\
\text { (Section 2.3) }\end{array}$ \\
\hline 0.00 & 0.010501 & 0.010422 & 0.010413 & $0.84 \%$ & $0.086 \%$ \\
0.10 & 0.015238 & 0.015246 & 0.015230 & $0.053 \%$ & $0.11 \%$ \\
0.15 & 0.021530 & 0.021465 & 0.021430 & $0.42 \%$ & $0.12 \%$ \\
0.20 & 0.030564 & 0.030435 & 0.030396 & $0.55 \%$ & $0.13 \%$ \\
0.25 & 0.042329 & 0.042249 & 0.042192 & $0.32 \%$ & $0.13 \%$ \\
0.30 & 0.056922 & 0.056854 & 0.056776 & $0.25 \%$ & $0.14 \%$ \\
0.35 & 0.074221 & 0.074001 & 0.073900 & $0.43 \%$ & $0.14 \%$ \\
0.40 & 0.093561 & 0.093249 & 0.093122 & $0.47 \%$ & $0.14 \%$ \\
0.45 & 0.11408 & 0.11399 & 0.11384 & $0.21 \%$ & $0.13 \%$ \\
0.50 & 0.13705 & 0.13605 & 0.13534 & $1.26 \%$ & $0.53 \%$ \\
\hline
\end{tabular}

(Section 2.2) - single region approach with constant coefficients eigenvalue problem. (Section 2.3) - single region approach with variable coefficients eigenvalue problem.

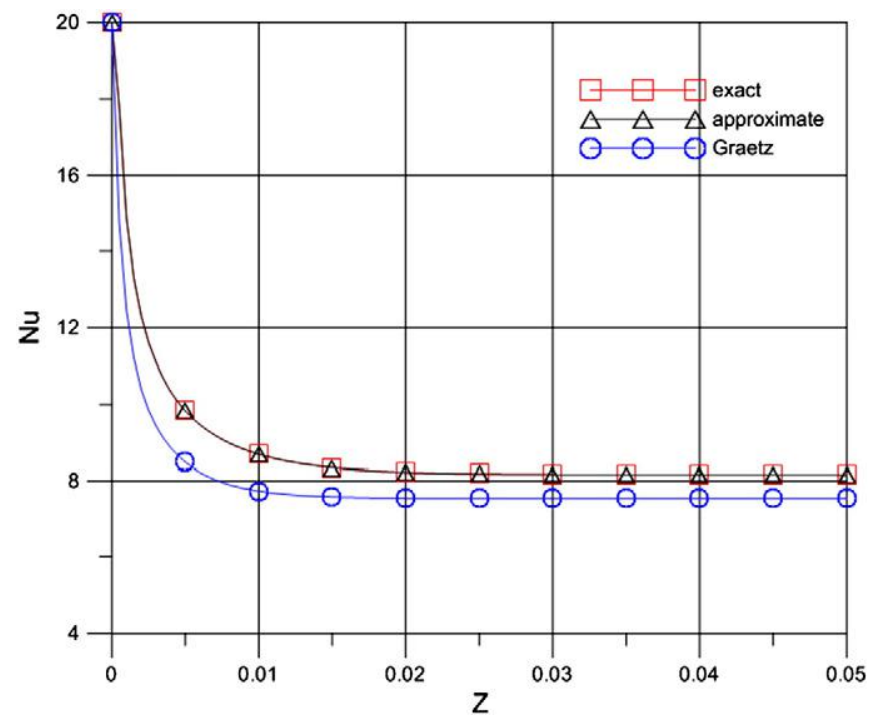

Fig. 5. Nusselt number calculated from the approximate (Sections 2.2 and 2.3) and the exact solutions, compared to the classical Graetz problem with prescribed wall temperature.

\section{References}

[1] G.L. Morini, Single-Phase Convective Heat Transfer in Microchannels: a Review of Experimental Results, International Journal of Thermal Sciences 43 (2004) 631-651.

[2] Y. Yener, S. Kakaç, M. Avelino, T. Okutucu, Single-Phase Forced Convection in Microchannels - a State-of-the-art Review", in: S. Kakaç, L.L. Vasiliev, Y. Bayazitoglu, Y. Yener (Eds.), Microscale Heat Transfer - Fundamentals and Applications, Kluwer Academic Publishers, The Netherlands, NATO ASI Series, 2005, pp. 1-24.

[3] S. Yu, T.A. Ameel, Slip Flow Heat Transfer in Rectangular Microchannels, International Journal of Heat and Mass Transfer 44 (2001) 4225-4234.

[4] G. Tunc, Y. Bayazitoglu, Heat Transfer in Microtubes with Viscous Dissipation, International Journal of Heat and Mass Transfer 44 (2001) 2395-2403.

[5] G. Tunc, Y. Bayazitoglu, Heat Transfer in Rectangular Microchannels, International Journal of Heat and Mass Transfer 45 (2002) 765-773.

[6] M.D. Mikhailov, R.M. Cotta, Mixed Symbolic-Numerical Computation of Convective Heat Transfer with Slip Flow in Microchannels, International Communications in Heat and Mass Transfer 32 (2005) 341-348.

[7] R.M. Cotta, S. Kakaç, M.D. Mikhailov, F.V. Castellões, C.R. Cardoso, Transient Flow and Thermal Analysis in Microfluidics, in: S. Kakaç, L.L. Vasiliev, Y. Bayazitoglu, Y. Yener (Eds.), Microscale Heat Transfer - Fundamentals and Applications, NATO ASI Series, Kluwer Academic Publishers, The Netherlands, 2005, pp. 175-196.

[8] F.V. Castellões, R.M. Cotta, Analysis of Transient and Periodic Convection in Microchannels via Integral Transforms, Progress in Computational Fluid Dynamics 6 (6) (2006) 321-326.

[9] F.V. Castellões, C.R. Cardoso, P. Couto, R.M. Cotta, Transient Analysis of Slip Flow and Heat Transfer in Microchannels, Heat Transfer Engineering 28 (6) (2007) 549-558.

[10] F.V. Castellões, R.M. Cotta, Heat Transfer Enhancement in Smooth and Corrugated Microchannels, Proc. of the 7th Minsk Int. Seminar on Heat Pipes, Heat Pumps, Refrigerators, Invited Lecture, Minsk, Belarus, 8-11 September 2008, 2008. 
[11] F.V. Castellões, J.N.N. Quaresma, R.M. Cotta, Convective Heat Transfer Enhancement in Low Reynolds Number Flows with Wavy Walls, International Journal of Heat and Mass Transfer 53 (2010) 2022-2034.

[12] J.S. Nunes, R.M. Cotta, M. Avelino, S. Kakaç, Conjugated Heat Transfer in Microchannels, in: S. Kakaç, B. Kosoy, A. Pramuanjaroenkij (Eds.), Microfluidics Based Microsystems: Fundamentals and Applications, NATO Science for Peace and Security Series A: Chemistry and Biology, v.1, 2010, pp. 61-82.

[13] G. Maranzana, I. Perry, D. Maillet, Mini and Microchannels: Influence of Axial Conduction in the Walls, International Journal of Heat and Mass Transfer 47 (2004) 3993-4004

[14] R.O.C. Guedes, R.M. Cotta, N.C.L. Brum, Heat Transfer in Laminar Tube Flow with Wall Axial Conduction Effects, J, Thermophysics \& Heat Transfer 5 (4) (1991) 508-513.

[15] R.M. Cotta, Hybrid numerical-analytical approach to nonlinear diffusion problems, Numerical Heat Transfer Part B 127 (1990) 217-226.

[16] R.M. Cotta, Integral Transforms in Computational Heat and Fluid Flow, CRC Press, 1993.

[17] R.M. Cotta, Benchmark results in computational heat and fluid flow: the integral transform method, International Journal of Heat and Mass Transfer (Invited Paper) 37 (Suppl. 1) (1994) 381-394.
[18] R.M. Cotta, M.D. Mikhailov, Heat Conduction: Lumped Analysis, Integral Transforms, Symbolic Computation, Wiley-Interscience, Chichester, UK, 1997.

[19] R.M. Cotta, The Integral Transform Method in Thermal and Fluids Sciences and Engineering, Begell House, New York, 1998.

[20] R.M. Cotta, M.D. Mikhailov, Hybrid methods and symbolic computations, in: W.J Minkowycz, E.M. Sparrow, J.Y. Murthy (Eds.), Handbook of Numerical Heat Transfer, 2nd edition, John Wiley, New York, 2006, (Chapter 16).

[21] C.P. Naveira Cotta, R.M. Cotta, H.R.B. Orlande, O. Fudym, Eigenfunction expansions for transient diffusion in heterogeneous media, International Journal of Heat and Mass Transfer 52 (2009) 5029-5039.

[22] M.D. Mikhailov, M.N. Ozisik, Unified Analysis and Solution of Heat and Mass Diffusion, John Wiley, 1984.

[23] L.A. Sphaier, R.M. Cotta, C.P. Naveira-Cotta, J.N.N. Quaresma, The UNIT algorithm for solving one-dimensional convection-diffusion problems via integral transforms, International Communications in Heat and Mass Transfer 38 (2011) $565-571$.

[24] J.V.C. Ayres, D.C. Knupp, C.P. Naveira-Cotta, L.O.S. Ferreira, R.M. Cotta, (2011) Evaluation of Infrared Thermography Experimental Analysis of a Single MicroChannel Heat Spreader, 21st International Congress of Mechanical Engineering, COBEM-2011, ABCM, Natal, RN, Brazil, October, 2011. 